Volume 8. No. 10, October 2020

International Journal of Emerging Trends in Engineering Research

Available Online at http://www.warse.org/IJETER/static/pdf/file/ijeter048102020.pdf

https://doi.org/10.30534/ijeter/2020/048102020

\title{
A Framework to Detect the Atrial Depolarization Level for the Diagnosis of Atrial Fibrillation
}

\author{
Avvaru Srinivasulu ${ }^{1 *}$, Y Dileep Kumar ${ }^{2}$, Praveen Kumar Medarametla ${ }^{3}$, and T V S Gowtham Prasad ${ }^{4}$ \\ ${ }^{1}$ Department of Electrical, Electronics and Communication Engineering, GITAM (Deemed to be University), \\ Bangalore campus, India. Email: sreesri.avvaru@ gmail.com \\ ${ }^{2}$ Bio-Signal Research Center, Department of Electronics, and Instrumentation Engineering, Sree Vidyanikethan, \\ Engineering College, Tirupati, India. Email: y.dileepkumar@yahoo.com \\ ${ }^{3}$ School of Electrical Engineering, VIT University, Vellore, India. Email: praveen.m@ vit.ac.in \\ ${ }^{4}$ Department of Electronics, and Communication Engineering, Sree Vidyanikethan, Engineering College, \\ Tirupati, India. Email: tvsgowtham@gmail.com
}

\begin{abstract}
The electrical depolarization of the atrium indicates the P-wave in Electrocardiogram. P-wave abnormality infers the various diseases like pulmonary hypertension, coropulmonale from chronic respiratory disease, and multifocal atrial tachycardia. The diseases like atrial fibrillation, atrial flutter, and sinoatrial arrest cause missing P-wave. Therefore, it is needed to detect the P-wave presence in the ECG signal for the diagnosis of AF patients. Therefore, in this paper, a framework was proposed to find the absence of P-wave in each beat of ECG. The ECG data is collected from the Atrial fibrillation database. The morphological features of each beat were extracted, and the most significant features were identified using PCA. The reduced feature vector was applied to the KNN for the detection of P-waves. Finally, it is concluded that the P-waves were detected by using the proposed framework with an accuracy of $96.6 \%$ without PCA feature reduction and $98.9 \%$ with PCA feature reduction.
\end{abstract}

Key words: Atrial Depolarization Level, P-wave detection, Atrial Conduction, Principle Component Analysis (PCA), K-Nearest Neighborhood (KNN).

\section{INTRODUCTION}

Atrial depolarization is the contraction of the left and right atrium, initiated by the Sino-Atrial (SA) node [1]-[2]. The depolarization of the atrium indicates the P-wave in the ECG signal. The P-wave occurs before the atrium contraction and pumping blood to the ventricles [3]. The regularity of P-wave occurrence depends on the activation of the SA node [4]. If P-amplitude $<0.15 \mathrm{mV}$ [5] and P-duration < 20msec [6], $\mathrm{P}$-wave is said to be absent [7]. The absence of $\mathrm{P}$-wave in AF patients is as shown in Figure 1. The irregularity in RR interval and regular absence of $\mathrm{P}$-wave infers the Atrial Fibrillation (AF) [8]. Therefore, the p-wave detection is essential in the diagnosis of AF patients. AF occurs when the atria do not pump the blood regularly and effectively to ventricles. This leads to clot the blood in the arteries, which causes heart stroke and heart failure.

Further, untreated AF leads to an increase in the risk level of heart stroke [9]. Therefore, the atrial depolarization level (P-wave) is a good indicator for the diagnosis of AF. The remaining paper was presented as follows. The related literature of $\mathrm{P}$-wave detection was reported in section II. The proposed framework was explained in section III. The results were reported and discussed in section IV. Finally, the paper was concluded with a summary in section $\mathrm{V}$.

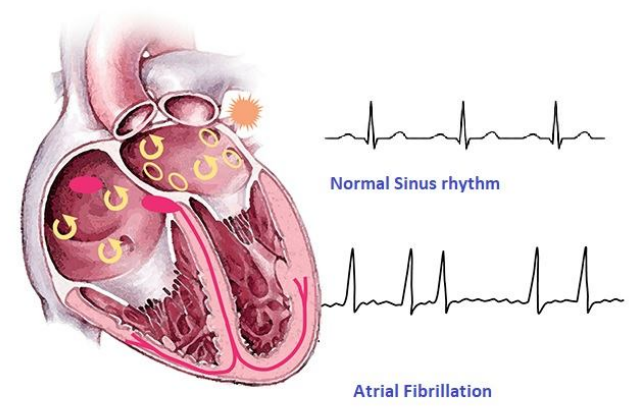

Figure 1. Atrial Fibrillation ECG waveform

\section{RELATED LITERATURE}

The P-wave detection is not as easy as the QRS complex detection due to the smaller amplitude of P-wave. There is some literature available where previous researchers developed different approaches to detect the P-waves in ECG. In [10], the p-wave was detected by finding the local minimum and local maximum points using parallel delta modulator architecture. They achieved an accuracy of $91 \%$ 
with datasets taken from the QT database. In [11], the researcher found the QRS complex using ensemble empirical mode decomposition. The QRS complex was removed to separate the P-wave. They achieved a sensitivity of $99.96 \%$ and positive predictivity of $99.47 \%$ with the QT database. In [7], QRS-T waves were detected by the Teager Energy operator, and P-wave was found by the slope criterion. They achieved a sensitivity of $96.47 \%$ using the CSE database. In [12], P-waves were found by extracting unique spectral properties form raw and nonlinearly synthesized high resolution pseudo-spectral multi-resonance signatures of the p-wave taken from the MIT-BIH database. The placement of electrodes is crucial to measure the significant level of P-wave in ECG, which was addressed in [13]. They investigated the effect of misplacement in p-lead to find the p-wave by calculating the P/QRST RMS ratio. They found that the median p-wave RMS amplitude was $72 \mu \mathrm{V}$ with $\mathrm{p}<0.001$. In previous literature works, machine learning algorithms such as ANN, decision tree [14], Support Vector Machine (SVM) [15] and deep learning Recurrent Neural Network algorithms [16] were applied physiological data for various biomedical applications. Therefore, in this study, we have applied K-Nearest Neighbourhood (KNN), SVM and Decision Tree algorithms on Atrial Fibrillation (AF) datasets to detect the atrial depolarization level which helps to assist the clinical diagnosis of AF patients.

\section{PROPOSED FRAMEWORK}

The P-wave detection methods reported in the literature was depended on the accuracy of QRS detection and the removal of QRST waves from the ECG signal. Therefore, in this paper, a framework was proposed to separate and detect significant P-waves from an ECG signal as shown in Figure 2.

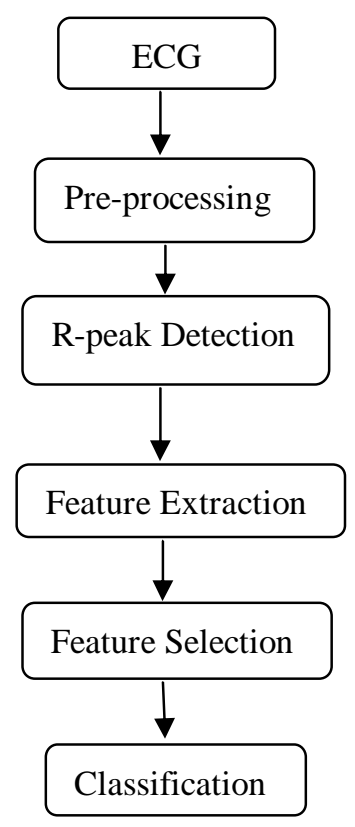

Figure 2. Flow chart of the proposed framework
In the proposed framework, the ECG signals were collected form Atrial Fibrillation Database [17]. A total of 31 records of one minute were used for the current study. The sampling rate of the ECG signal is 250 samples per second. The ECG signal was pre-processed by using a smoothing filter and wavelet transform (WT). The glitches were removed by the smoothing filter [18]. The baseline drift was removed by WT [19]. The 8th level approximation coefficient was reconstructed from 10th level decomposition with db6 mother wavelet. The reconstructed signal was subtracted from raw ECG to remove the baseline drift. The R-peaks were found by using the Pan-Tompkins algorithm [20]. A segment of R-0.2sec to R-0.09sec was separated and extracted the morphological features of all 31 samples. The morphological features from each beat are measured. The feature vector of 31 features was reduced by using the PCA method [21]. The reduced feature vector was applied to the KNN classifier to detect the significant $\mathrm{P}$-wave presence. The sample of $\mathrm{P}$-wave presence and absence was shown in Figure 3.
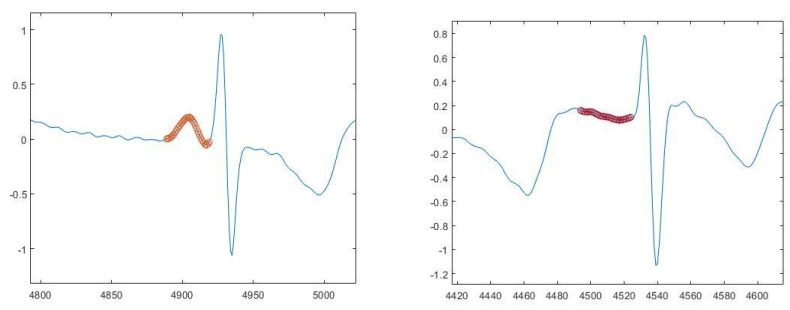

Figure 3. P-wave presence (left) and absence (right) in ECG

\section{RESULTS}

The ECG was collected from the Atrial Fibrillation database. A segment of a record was as shown in Figure 4. Glitches from ECG were removed using a moving average filter, which can be observed in Figure 4 (middle).

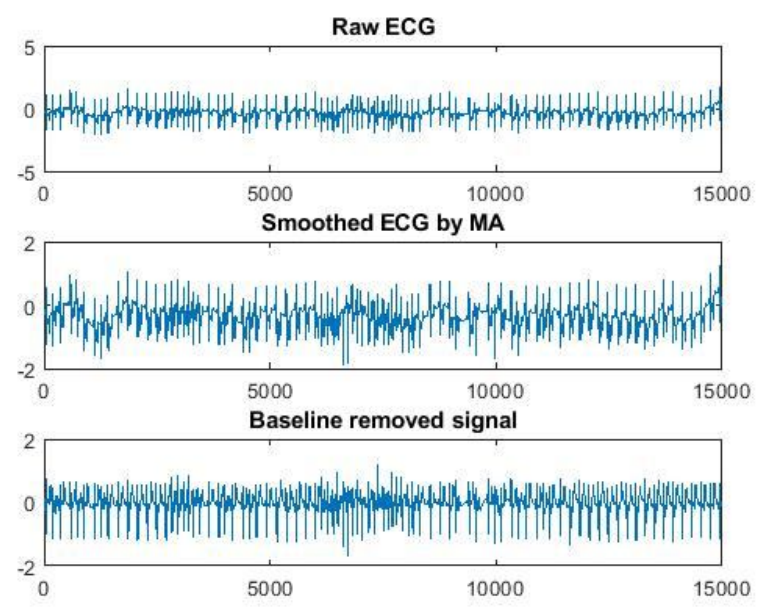

Figure 4. Raw and Filtered ECG signals (record \#04015) 


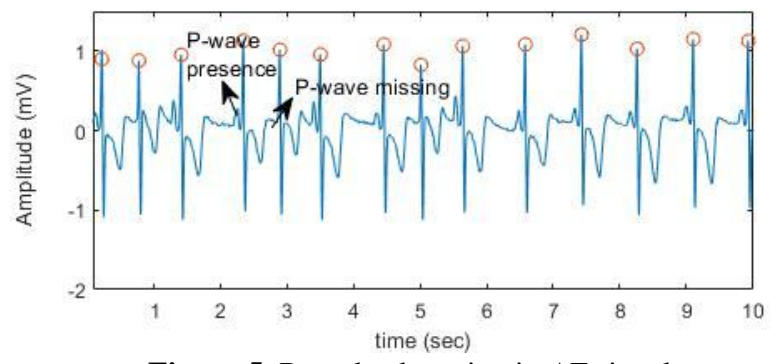

Figure 5. R-peaks detection in AF signal

The baseline drift was removed effectively using WT observed from Figure 4 (bottom). The R-peaks detected by the Pan-Tompkins algorithm, with 100\% accuracy were shown in Figure 5. In normal ECG, the P-wave occurs $0.12-0.20$ sec before every R-peak occurrence. Since the $\mathrm{P}$-wave duration is $80 \mathrm{msec}$, the morphological features of this segment were extracted to find out the P-wave significance, as shown in Figure 6.

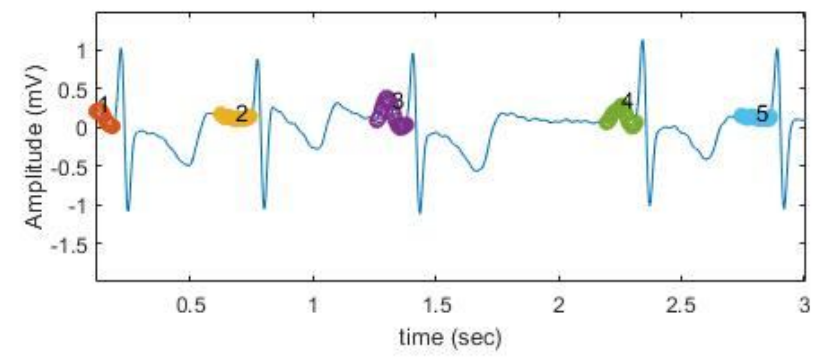

Figure 6. P-wave segment detection in AF signal

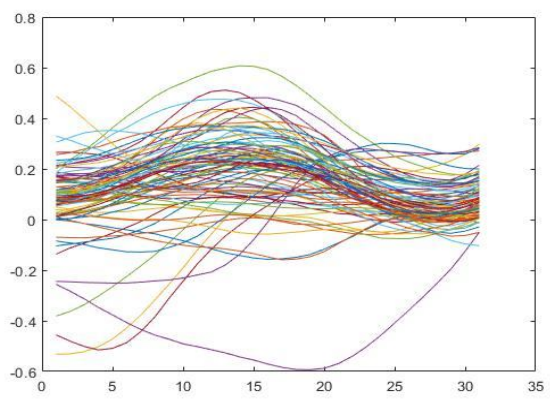

Figure 7. P-wave segments in AF signal

Even the P-wave presence can be detected by its amplitude $(>0.15 \mathrm{mV})$, it is difficult to find the amplitude in abnormal cases, as shown in Figure 7. Also, due to signal pre-processing, the amplitude will not be the same as the raw ECG signal. Therefore, in this proposed framework, the extracted morphological features were applied to the KNN classifier to detect the significant P-waves. In other words, abnormal P-waves have been separated by the proposed framework. Due to the feature vector size was 31 , the size was optimized to 3 by using PCA and tested the accuracy of the KNN classifier. The performance of the proposed work was evaluated in terms of accuracy, sensitivity, specificity, and positive predictivity given by the equations (1) - (4).

$$
\text { sonsitivity }(\%)=\frac{T_{F}}{F^{*}+F} \times 100
$$

$$
\begin{aligned}
& \text { specificity }(\%)=\frac{W N}{T H+F E} \times 100 \\
& \text { postivepredictivity }(\%)=\frac{W}{W+F} \times 100
\end{aligned}
$$

(3)

$$
\text { Accuray }(\%)=\frac{T^{*}+T W}{F^{*}+E^{2}+F N+F N} \times 100
$$

(4)

Where, TP is the true $\mathrm{p}$-wave presence

$\mathrm{TN}$ is the true $\mathrm{p}$-wave missing

FP is the false $\mathrm{p}$-wave presence

$\mathrm{FN}$ is the false $\mathrm{p}$-wave missing

Table1. Classification results before feature reduction

\begin{tabular}{|l|l|l|l|l|}
\hline Classifier & Accuracy & Se & Sp & P+ \\
\hline Decision Tree & 95.4 & 94.64 & 96.77 & 98.15 \\
\hline SVM & $\mathbf{9 6 . 6}$ & $\mathbf{9 6 . 4 3}$ & $\mathbf{9 6 . 7 7}$ & $\mathbf{9 8 . 1 8}$ \\
\hline KNN & $\mathbf{9 6 . 6}$ & $\mathbf{9 6 . 4 3}$ & $\mathbf{9 6 . 7 7}$ & $\mathbf{9 8 . 1 8}$ \\
\hline
\end{tabular}

Table 2. Classification results after feature reduction

\begin{tabular}{|l|l|l|l|l|}
\hline Classifier & Accuracy & $\mathrm{Se}$ & $\mathrm{Sp}$ & $\mathrm{P}+$ \\
\hline decision tree & 88.5 & 92.86 & 80.65 & 89.66 \\
\hline SVM & 96.6 & 96.43 & 96.77 & 98.18 \\
\hline KNN & $\mathbf{9 8 . 9}$ & $\mathbf{1 0 0 . 0 0}$ & $\mathbf{9 6 . 7 7}$ & $\mathbf{9 8 . 2 5}$ \\
\hline
\end{tabular}

Table 3. Comparison of results with literature

\begin{tabular}{|l|l|l|l|l|l|}
\hline Ref & Database & Accuracy & Se & Sp & P+ \\
\hline$[10]$ & QT database & 91 & - & - & - \\
\hline$[11]$ & QT database & - & 99.96 & - & 99.47 \\
\hline$[7]$ & $\begin{array}{l}\text { CSE } \\
\text { database }\end{array}$ & - & 96.47 & - & - \\
\hline Proposed & AF database & 98.9 & 100 & 96.77 & 98.25 \\
\hline
\end{tabular}

The classification accuracy of the KNN classifier with and without feature reduction was presented in Tables $1 \& 2$. It was observed that the highest accuracy of $96.6 \%$, the sensitivity of $96.43 \%$, Specificity of $96.77 \%$, and positive predictivity of $98.18 \%$ was obtained for both SVM and KNN classifiers without feature reduction. The highest accuracy of $98.9 \%$, the sensitivity of $100 \%$, Specificity of 96.77 , and positive predictivity of $98.25 \%$ was obtained using the KNN classifier with feature reduction. Finally, the detection accuracy of the proposed work was compared with the previous literature. It was observed that the proposed framework exhibited better performance compared to the literature, as shown in Table 3.

\section{CONCLUSION}

The Atrial Fibrillation is a severe cardiac disease which further causes heart stroke. In this regard, a framework was proposed in this paper to detect the P-wave presence (atrial 
depolarization). The detection of $\mathrm{P}$-wave was done by extracting morphological features from each beat. The P-wave presence and absence beats were separated by using the KNN classifier. The proposed framework classified the P-wave absence with an accuracy of $96.6 \%$. By applying the PCA feature reduction, the accuracy was increased to $98.9 \%$. Also, the proposed framework detected the P-waves with accuracy compared to the literature.

\section{REFERENCES}

[1] Kashou, A.H., Basit, H. and Chhabra, L. Physiology, sinoatrial node (SA node), 2019.

[2] Bikkuri, P. and Srinivasulu, A., 2020. Design Issues of Doppler Radar For Heart Rate Sensing-A Systematic Review. Biomedical Engineering: Applications, Basis and Communications, p.2050038. 2020.

[3] Boineau, J.P., Schuessler, R.B., Mooney, C.R., Wylds, A.C., Miller, C.B., Hudson, R.D., Borremans, J.M. and Brockus, C. Multicentric origin of the atrial depolarization wave: the pacemaker complex. Relation to dynamics of atrial conduction, P-wave changes and heart rate control. Circulation, 58(6), pp.1036-1048, 1978.

[4] Wagner, G.S. and Lim, T.H. Interpretation of the normal electrocardiogram. Marriott's practical electrocardiography, 9, p.50, 2008.

[5] Bai, V.T. Real time portable telemedicine system for cardiac patient. In 2009 International Conference on Future BioMedical Information Engineering (FBIE) (pp. 57-60). IEEE, 2009.

[6] Sasaki, R., Sugisawa, K., Tani, H., Kitagaki, Y., Nishian, K. and Tateishi, J. Correlation Between Initial Potentials on a Signal-Averaged P-Wave and Indice of Electrophysiologic Measurements in the Right Atrium. Japanese circulation journal, 62(4), pp.279-283, 1998.

[7] Castrillón, R., Pérez, J.J. and Andrade-Caicedo, H. Electrical performance of PEDOT: PSS-based textile electrodes for wearable ECG monitoring: a comparative study. Biomedical engineering online, 17(1), p.38, 2018.

[8] Hermida, J.S., Kubala, M., Lescure, F.X., Delonca, J., Clerc, J., Otmani, A., Jarry, G. and Rey, J.L. Atrial septal pacing to prevent atrial fibrillation in patients with sinus node dysfunction: results of a randomized controlled study. American heart journal, 148(2), pp.312-317, 2004.

[9] Petersen, P. and Godtfredsen, J. Risk factors for stroke in chronic atrial fibrillation. European heart journal, 9(3), pp.291-294, 1998.

[10] Vallejo, M., Márquez, M.F., Borja-Aburto, V.H., Cárdenas, M. and Hermosillo, A.G.. Age, body mass index, and menstrual cycle influence young women's heart rate variability. Clinical Autonomic Research, 15(4), pp.292-298, 2005.
[11]Di Rienzo, M., Rizzo, F., Parati, G., Ferratini, M., Brambilla, G. and Castiglioni, P. A textile-based wearable system for vital sign monitoring: applicability in cardiac patients. In Computers in Cardiology, 2005 (pp. 699-701). IEEE, 2005.

[12] Di Rienzo, M., Racca, V., Rizzo, F., Bordoni, B., Parati, G., Castiglioni, P., Meriggi, P. and Ferratini, M. Evaluation of a textile-based wearable system for the electrocardiogram monitoring in cardiac patients. Europace, 15(4), pp.607-612, 2012.

[13] Koenig, J., Jarczok, M.N., Warth, M., Ellis, R.J., Bach, C., Hillecke, T.K. and Thayer, J.F. Body mass index is related to autonomic nervous system activity as measured by heart rate variability-a replication using short term measurements. The journal of nutrition, health \& aging, 18(3), pp.300-302, 2014.

[14]A. Vijaya Lakshmi, K. V. T. Nagendra Babu, M. Sree Ram Deepak, A. Sai Kumar, G. V. P. Chandra Sekhar Yadav, V. Gopi Tilak, V. S. Ghali. A Machine Learning based Approach for Defect Detection and Characterization in Non-Linear Frequency Modulated Thermal Wave Imaging. International Journal of Emerging Trends in Engineering Research. Volume 7, No. 11, pp 517-522, November 2019.

[15] N. Chandra Sekhar Reddy, Purna Chandra Rao Vemuri, A. Govardhan. An Empirical Study on Support Vector Machines for Intrusion Detection. International Journal of Emerging Trends in Engineering Research. Volume 7, No. 10, pp 383-387, October 2019.

[16] Praveen Kumar Kollu1, R. Satya Prasad. Intrusion Detection System Using Recurrent Neural Networks and Attention Mechanism. International Journal of Emerging Trends in Engineering Research. Volume 7, No. 8, pp 178-182, August 2019.

[17] Moody GB, Mark RG. A new method for detecting atrial fibrillation using $\mathbf{R}-\mathbf{R}$ intervals. Computers in Cardiology. 10:227-230, 1983.

[18] Pandey, V. and Giri, V.K. High frequency noise removal from ECG using moving average filters. In 2016 International Conference on Emerging Trends in Electrical Electronics \& Sustainable Energy Systems (ICETEESES) (pp. 191-195). IEEE, 2016.

[19] Tinati, M.A. and Mozaffary, B. A wavelet packets approach to electrocardiograph baseline drift cancellation. International journal of biomedical imaging, 2006.

[20] Avvaru Srinivasulu, Srinivasa Rao Lam. Cardiac Episode Detection and Classification - A Systematic Review. International Journal of Recent Technology and Engineering, Volume-7, Issue-ICETESM, pp 35-42. 2019

[21] Avvaru Srinivasulu, Y Dileep Kumar. Effect of PCA Feature Reduction on Ventricular Ectopic Beat Classification. International Journal of Engineering and Advanced Technology, Volume-9 Issue-1S5. Pp 29-32, 2019. 